\title{
An innovative approach to the design of multi-bolted joint assemblies in the uncertain early stages
}

\author{
T. Dericquebourg ${ }^{1}$, D. Lenoir ${ }^{2}$, F. Debarle ${ }^{1} \&$ L. Jézéquel $^{2}$ \\ ${ }^{I}$ PSA Peugeot Citroën, Centre Technique de La Garenne-Colombes, \\ France \\ ${ }^{2}$ Laboratoire de Tribologie et Dynamique des Systèmes, \\ Ecole Centrale de Lyon/Université de Lyon, France
}

\begin{abstract}
In the automotive industry, the optimization of the preliminary stages is a major asset for reducing the time of the design process. So, a new approach for setting up the optimal configuration of a bolted joints assembly has been developed. The aim is to efficiently fix the main characteristics of bolted joint assemblies in a particularly uncertain context. Indeed, in preliminary design, choices are made with little information and many indeterminations, but these should be relevant whatever the following steps of the design process may be. The methodology developed is based on simplified models included in a strategy consisting of testing configurations to choose the best one. In order to scan the design space well, an optimized Latin hypercube strategy is used. Then, algorithms have been developed to find clusters of good items and of unacceptable ones. These clusters are then used to specify a robust solution or to avoid incorrect configurations. An application was conducted on an automotive mounting. The reduced time needed to obtain useful results really contributes to shortening the design process duration and the approach allows one to control the risks of the process uncertainties.
\end{abstract}

Keywords: mechanical design, preliminary design, bolted joints, uncertain context, modelling. 


\section{Introduction}

Time is one of the main industrial constraints for manufacturers, who are continually seeking to improve the efficiency of the first stages of the design process. The decisions made in the preliminary stages considerably affect the following ones and, consequently, the due time criteria. This aspect is predominant in the automotive industry since time reduction is an asset for efficient performance in the current competitive market. This aspect is reflected in all the fields of the car manufacturing hierarchy, in the acoustical domain for example, where engineers try to quickly assess the acoustical performances of design alternatives using meta-models to improve space allocations [1]. It is also particularly true in bolted adjoint assemblies. Such assemblies are widespread in the automotive industries since they are very practical for setting up removable junctions between the subassemblies of the body structures or unit designs. Designers always seek to specify the number, the position or the main characteristics of bolted joints quickly and efficiently. In this framework, it may be too risky to rely only on designer experience and on simple empirical formulas based on simplified hypotheses. Too basic an approach may well be of little help since it may not take into consideration the most significant parameters and complex constraints for some problems.

The object of this paper is to present a new approach to assist designers in making the most appropriate choices in the first stages of the bolted joints definition process. This paper outlines the design of screwed assemblies, and thus the optimization of the main parameters at the higher level of the design cycle to satisfy the maximum mechanical criteria. The study was conducted on industrial cases and the methodology has been developed particularly on mountings made up of subassemblies linking the engine to the body shell with screwed joints. An example of mounting can be seen in figure 1 - this is a common bolted assembly in car industries.

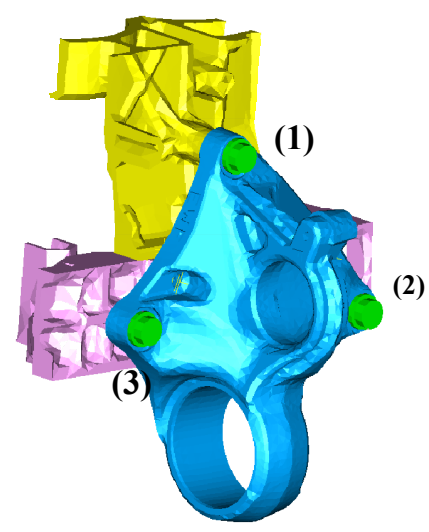

Figure 1: Example of a mounting fastened to an engine crankcase with three bolts. 


\section{Specific context}

The specific context of this study has a significant impact on the way to settle the problem. Indeed, the methodology has been developed in order to be used in the early stages of the design process. In these early stages, the context is very uncertain because almost no information is known, or if information is given, it is not clear or is prone to alteration. Moreover, the decisions made in the first steps of the design process can have a real impact on the following design steps and so on the design cycle time.

In preliminary design, the aim of multi-bolted joint assembly predimensioning is to determine a good configuration that will fulfil several requirements. A configuration of a bolted joints assembly is defined by the main characteristics of the assembly and the main requirements are essentially the screw strength, preventing mating of parts in contact, preventing sliding and preventing detachment. However, in this context, the uncertainties are multiple and of several kinds. The aim of the methodology is to suggest some solutions that are able to fulfil the needs and give some accurate information about the capabilities of the solutions to satisfy the criteria in order to help the designers to efficiently make the good decisions as early as possible in the process.

\subsection{Uncertain input data}

In preliminary design, input data are not well defined. Indeed, at the first stages of the design process, the different data are estimated but not accurately calculated: for example, the loads or some geometrical characteristics of the surroundings parts cannot be precisely known at this step, but can only be estimated thanks to previous conceptions. As all the external characteristics of a part, such as loads or common contacts, cannot be accurately identified, it is difficult to correctly design the assembly and to be sure that the created design will fit the needs. Moreover, while the part is not completely defined, it is not easy to determine if this part will fulfil the requirements to declare the part well designed or not. As a result, the main difficulty in this context is to be able to recommend a good solution that fits the needs when not all surrounding parameters are well known. This makes one of the main difficulties of the problem.

\subsection{Following design step uncertainties}

The first choices made during the beginning of the design process are very important because they can have high consequences on the following steps. Indeed, once the choice of a solution is made, all the following steps are consequences of this first choice. As a result, this first choice must absolutely be a good one in order to meet the deadlines of the design process and to avoid many loops between the design steps that are time consuming. In this context, for an adequate design, choices made at the first stages should be relevant during the following stages. This criterion is justified by the fact that the subassemblies may be designed and often developed in different departments and, therefore, by 
different engineers in different design offices. Thus the main parameters of an assembly, which have a real impact on the design of the subassemblies, have to be established as soon in the process as possible. The main difficulty in this context is to be sure that the solution will satisfy all the requirements as the design process goes along, because the consecutive steps are partly unpredictable.

\section{Methodology}

The methodology is based on simplified models that have been specifically developed. To develop a simplified model, several hypotheses have to be done and consequently restrict the validity field. In this case, the hypotheses retained to develop the simplified model are the following: the clamped part must be composed of a stiff centre area and flexible edges where the bolts are placed. Using the existing models found in the literature, such an assembly cannot be fully represented and thus cannot be efficiently designed. The best approach remains FE modelling. Unfortunately, this is heavy and time consuming, and thus inappropriate in an early phase of the design process. This is why the presented simplified modelling has been developed. Then, the methodology consists of using the simplified model in an iterative approach as a tool for choosing the optimal configuration for a bolted industrial assembly.

\subsection{General approach}

The principal objective of this methodology is to set up the main parameters and characteristics of bolted joint assemblies subjected to static or cyclic loads in normal and extreme operation. The mains parameters are the following:

- number of screws,

- position of bolted joints in the assembly,

- characteristics of the bolts (screw diameter, length, pre-load, etc...).

However, the main industrial constraints in the first stages of a design process are rapidity and simplicity. Hence, the methodology developed is based on simplified, easily executable models in the Matlab ${ }^{\circledR}$ environment. The key idea of the resolution procedure is to iterate on several potential configurations so as to find the one that best fits the various design criteria.

In this methodology, an architecture is defined by the positions and the number of screws. When geometrical characteristics (screw and local clamped part dimensions) and material properties are added, this defines a configuration (cf. figure 2). In the aim of investigating all the possible solutions, the designer must specify the following different input parameters:

- load values,

- location of the external loading,

- vacant geometric areas for screw implementation,

- vacant space for material filling.

Below, all possible configurations are investigated to set up stresses in the joints and bolts for each case. Finally, the results are compared and the optimal 
configuration is chosen by the designer. The described process highlights the necessity for very light, quick models.

\subsection{Simplified model}

The simplified model used in the methodology has been previously developed and presented in [2]. The simplified model is made up of two levels: a local level dedicated to describe the local behaviour of a bolted link and a global level dedicated to compute the forces repartition between the bolts.

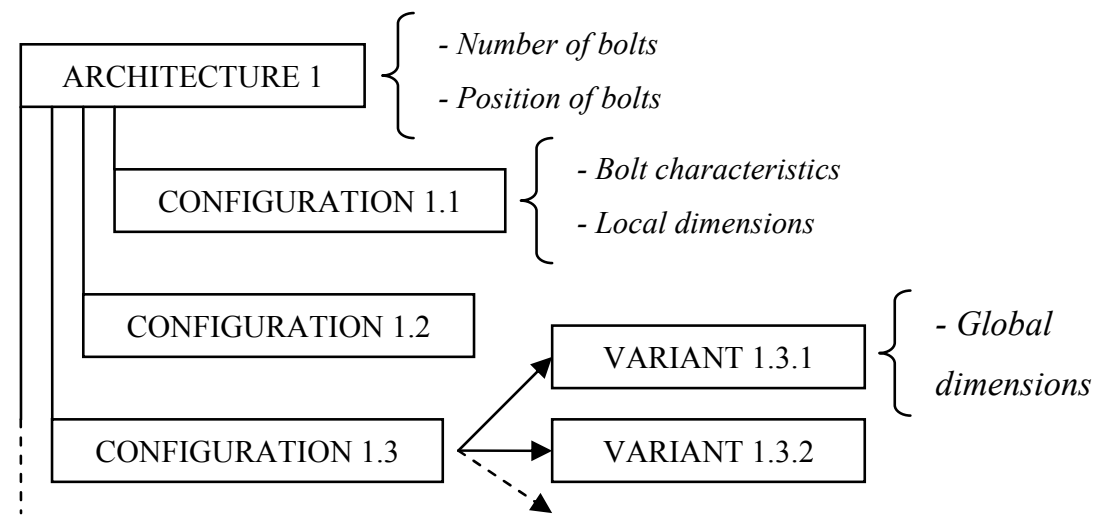

Figure 2: Solutions classification: a variant is completely defined and is based on the configuration of an architecture.

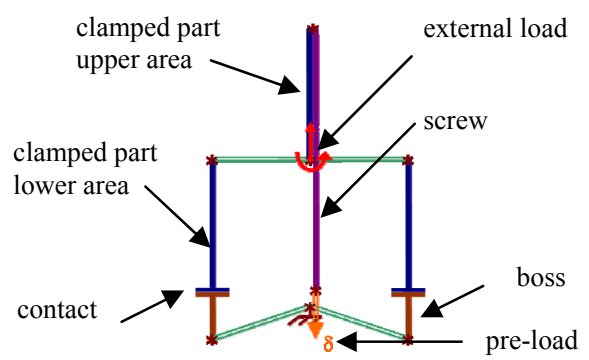

Figure 3: $\quad$ Simplified local model representation (longitudinal-section).

\subsubsection{Local level}

Concerning the local approach, a new model has been developed to overcome the limits of the existing models. The model can take complete or partial separation of the subassemblies into consideration under normal and extreme operating conditions. The local model represents a bolted joint consisting of a screw, its corresponding boss and the clamped subassembly (cf. Figure 3). It represents only the local zone of the parts, located near the screw. The inputs are the external load set up from the global model and the joint and screw geometric 
and physical characteristics. This model is essentially made up from simple finite elements like beam or rigid elements and node to node contact. The stiffness of the constitutive elements is fixed to physical values partially calculated thanks to the VDI [3] that are the most familiar references used in industrial design. But other adaptations have been made in order to be more predictive.

\subsubsection{Global level}

The main aim of the global level is to model the assembly using simple finite elements like rigid elements and beam elements; this model allows calculating the forces repartition between the joints that is function of the clamped part shape. This model can be described as a skeleton, since the different bolted joint centres are linked to the load application point by rigid and beam elements: rigid elements are used to model the stiff areas of a subassembly whereas beam elements are used to describe the flexible zones (cf. Figure 4). The characteristics of beam elements are established to fit the shape and stiffness of the subassemblies. Material properties are the same as in the real subassembly.

\subsection{Solutions searching strategy}

Starting with the input data listed previously, a listing of different architectures can be constructed. The strategy consists then in trying these several architectures and for each, testing several configurations. The robustness of each configuration is then estimated thanks to the simplified model. All parameters, except the ones concerning the global shape of the clamped part, are fixed in a configuration. When these last parameters are fixed at a defined value, the entire simplified model is defined and called a variant (cf. Figure 2).

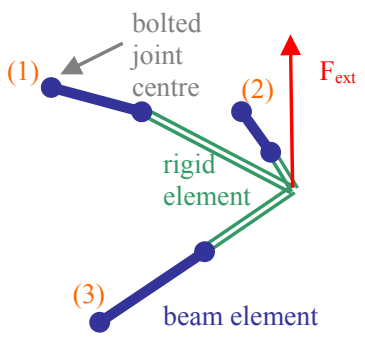

Figure 4: $\quad$ Equivalent global modelling of a 3-bolts assembly.

Table 1: $\quad$ Failure risks for every mechanical criterion and global failure risk for each configuration of the same architecture (illustration).

\begin{tabular}{|c|c|c|c|c|c|}
\hline Config. & Criterion 1 & Criterion 2 & $\ldots$ & Criterion $\mathrm{n}$ & Global criterion \\
\hline 1.1 & $10 \%$ & $40 \%$ & & $30 \%$ & $52 \%$ \\
\hline 1.2 & $12 \%$ & $16 \%$ & & $25 \%$ & $45 \%$ \\
\hline 1.3 & $8 \%$ & $23 \%$ & & $14 \%$ & $25 \%$ \\
\hline$\ldots$ & $\ldots$ & & & & \\
\hline
\end{tabular}


To estimate the viability of a configuration, some variants of the configuration are generated (at least a hundred) using an optimised Latin hypercube [4-6] tactic in order to well scan the design space. Each variant is calculated in order to check if the variant fulfil the mechanical criteria. Then a kind of failure risk can be given when calculating the ratio between the number of faulty variants and the number of tested variants. This calculation is done for each criterion of acceptance allowing determining which criterion is the most difficult to satisfy. Then, a table can be created to quickly show the results and the probabilities for each configuration of all possible architectures (cf. Table 1).

The simplified model allows testing all criteria that have to be checked in order to determine if a solution is acceptable or not. The main criteria tested are the followings:

- mechanical axial resistance,

- bending resistance,

- shear resistance,

- slip resistance,

- no detachment,

- fatigue resistance.

And finally, a global acceptance criterion is calculated, it represents the global failure risk for all the criteria together. This last criterion is not satisfied if just one of the other criteria is not satisfied.

This table is very useful to sort the different configurations in failure risk order: the lower the failure risk is, the easier the design process will be, but in the same time, the more expensive the assembly conception may be. On the other hand, if the failure risk is high, there will certainly be difficulties in the design process to obtain a viable assembly. The choice of the configuration must so be a compromise between the performance of the assembly (i.e. the capability of the assembly to satisfy the various mechanical criteria) and its cost (the design process cost as well as the production cost).

\subsection{Solutions gathering algorithm}

For each solution, useful information is given: the failure risk for each criterion. So if this risk is very weak, it is not too hazardous to choose this solution, but it will certainly be more expensive. It is more interesting to try to find a less expensive solution if it is possible to well specify it. Indeed, a solution can have a non neglecting failure risk but if you are able to well describe the parameters associations that lead to this failure or the ones that allow avoiding failure, it is much more interesting, because it is far possible that this solution will be cheaper. As said previously, the aim is to find the good compromise between the cost and the performance of the solution. And if specifications can be done to avoid failure areas, it will be possible to choose more risky solutions and obtain a cheaper solution with a risk control.

In order to find the design space areas to avoid or to use allowing specifications, two similar algorithms have been developed. One dedicated to find the clusters of good solutions and the other to find the clusters of bad solutions. 
The diagram in figure 6 exposes the algorithm structure and an example illustration can be shown on figure 5 . The initialisation of the algorithm consists in finding the first valid solution (1) that is the farthest of the bad ones:

$$
X_{1}^{\text {cluster }}=\max _{i \in I}\left(\min _{j \in J}\left(X_{i}^{o k}-X_{j}^{k o}\right)\right)
$$

where $\quad X_{1}^{\text {cluster }}$ barycentre of the cluster number 1

$X_{i}^{o k}$ variant number $i$ of the valid variants set

$X_{j}^{k o}$ variant number $j$ of the unacceptable variants set

$I$ set of valid variant indexes and $J$ set of unacceptable ones

Then, the iterative procedure of the algorithm works in that way: from the first good solution (1), the algorithm seeks for the nearest good solution (2), and with these two solutions a new cluster is created; a cluster is defined by the included solutions and the barycentre $X_{1}^{\text {cluster }}$.

$$
X_{1}^{\text {add }}=\min _{i \in I}\left(X_{i}^{\text {ok }}-X_{1}^{\text {cluster }}\right)
$$

where $X_{1}^{\text {add }}$ next variant to include in the cluster 1

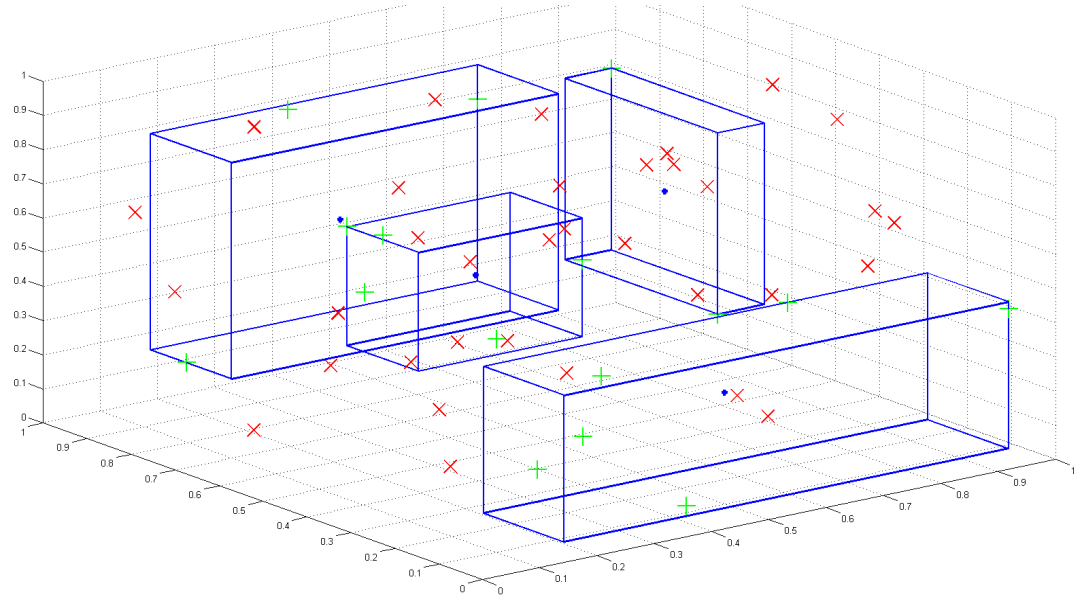

Figure 5: Example in 3-dimensions of good solution clusters extracted with the algorithm ( + : good solution, $\mathrm{x}:$ bad solution).

Then a test is made: if no bad solution is included inside the limits of the cluster, the cluster generation goes on by seeking again the nearest good solution (2) and so on..., else the cluster growth ends. So the nearest good solutions are iteratively included in the cluster until the cluster is going to encircle a bad solution. In this last case, the cluster growth ends and a new one is created by beginning with another good solution (3) determined like the first one (the farthest good solution from the bad ones) but here excluding the already grouped solutions. Then the growth of the cluster (4) is similarly performed. 


$$
\begin{gathered}
X_{k}^{\text {cluster }}=\max _{\substack{i \in I \\
i \notin C p, p<k}}\left(\min _{j \in J}\left(X_{i}^{o k}-X_{j}^{k o}\right)\right) \\
X_{k}^{\text {add }}=\min _{\substack{i \in I \\
i \notin C p, p \leq k}}\left(X_{i}^{\text {ok }}-X_{k}^{\text {cluster }}\right)
\end{gathered}
$$

where $\quad X_{k}^{\text {cluster }}$ barycentre of cluster number $k$

$X_{k}^{a d d}$ next variant to include in the cluster $p$

$C p$ set of variant indexes included in the cluster $p$

$k$ current cluster number

The second algorithm dedicated to group the defective variants has a similar working. Indeed, the structure is the same, except that the valid solutions are replaced by the bad ones and inversely.

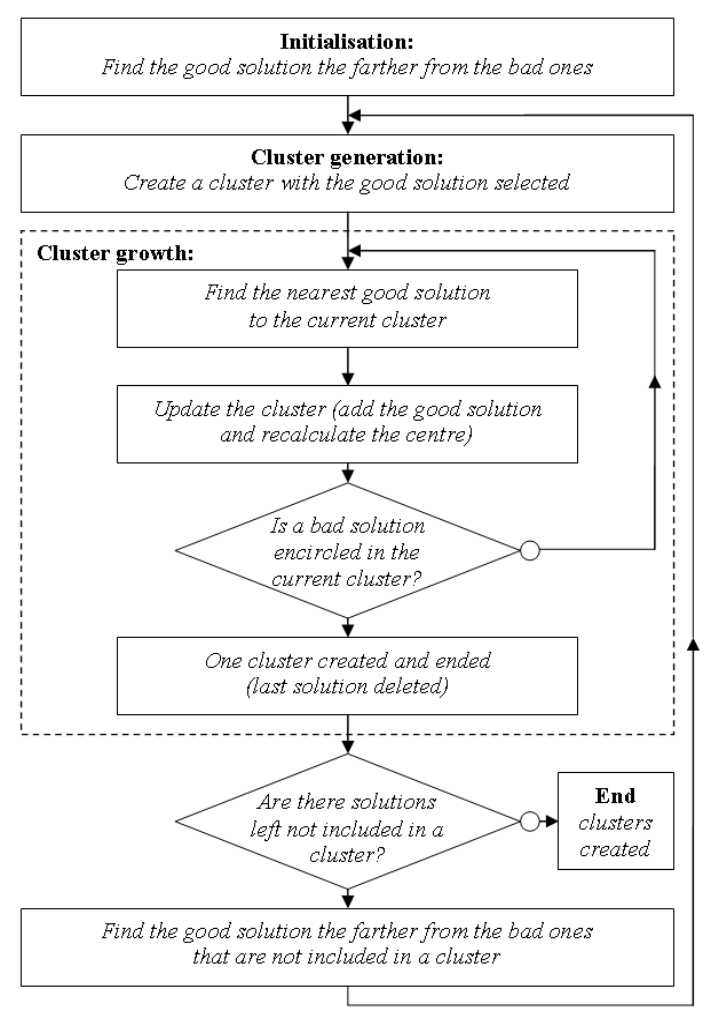

Figure 6: Algorithm structure. 


\section{Industrial application}

\subsection{Application case}

The methodology presented was directly applied to different mountings that are subassemblies linking the engine to the body shell of a car with bolted joints as shown on figure 1. The main function of these parts, fixed to the engine with several bolts, is to hold the latter in the car and to prevent its excessive movement. Generally, the mountings have similar shapes that satisfy the hypotheses of the simplified modelling: the core is often dense and rigid, while the edges are more flexible.

For the presented industrial application, two architectures, a 3 and a 4-screws one, are proposed and several configurations (four per architecture) are tested. The overall methodology takes only a few minutes to be performed. The objective of this application is to compare a 3 and a 4-screws solution and to precise the best value of the other parameters to design the optimal solution.

\subsection{Results obtained}

Tables of failure risks are given on figure 7. Two tables can be seen corresponding to the two architectures tested. A table gives the failure risks for several design criteria and a global failure probability for each configuration: a line corresponds to a configuration and a column corresponds to a criterion with the last one corresponding to the global risk.

The risk are here given by a proportional colour. It can be seen that the more safety solution seems to be the second configuration of the 4-screws architecture. What can be seen too is that the more restrictive criteria are the sixth and the eleventh ones that correspond respectively to the sliding resistance and the fatigue resistance.

3-screws architecture

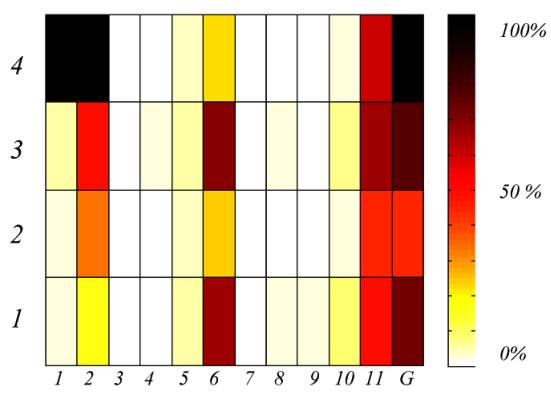

4-screws architecture

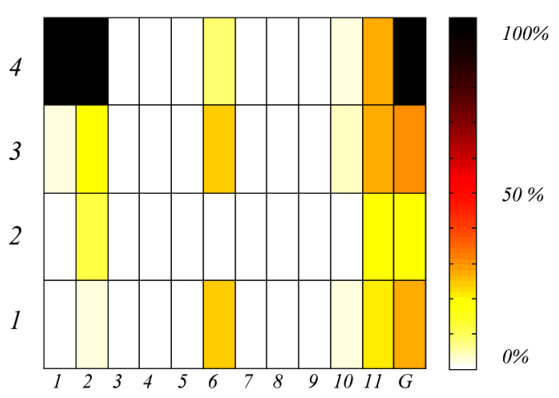

Figure 7: Tables of failure risks for two different architecture suggestions of a mounting (line $=$ configuration, column $=$ criterion). 
These first results help to have an idea of the potential good solutions by comparing the failure risks of the two suggested architectures, and moreover, inside each architecture, several configurations are balanced. Although this information is very useful, it is not enough to take the best decision. Indeed, it would be more interesting to know if the failure risks are concentrated in a limited area or if the failure risks are regularly distributed in the design space. A solution with weak failure risks could effectively be less interesting if the risks are uniformly distributed in the design space, because it will consequently be too difficult to efficiently avoid these unacceptable variants.

\subsection{Clusters generated}

The following table 2 shows the different clusters found thanks to the algorithms exposed previously. The computation has been made for just one level of quality of the bolts. The table gives the clusters of valid solutions and the clusters of faulty ones for every configuration.

In order to well use these results, the number and the size of every cluster have to be observed. Indeed, the easiest configuration to design is the one that has only valid variants. In this example, the configuration number 3 of the 3bolts architecture is rather simple to specify because, whereas the majority of the variants are unacceptable, the valid variants are distributed in only two clusters in the design space. Sometimes, like the third configuration of the 4-screws architecture, it is maybe preferable to specify the bad solutions clusters than the good ones because they are less numerous and so easier to take into consideration during the design phase.

This table gives to the designer additional information in the aim of making the best choice. Indeed, this output can be productively used to precise the good configurations: the designer can now take a more hazardous decision if he knows that the solution could be given with specifications in order to avoid the faulty parameters associations and could then certainly decrease the cost.

Table 2: Illustration of the clusters found by the algorithms.

\begin{tabular}{|c|c|c|c|c|c|c|}
\hline $\begin{array}{l}\stackrel{3}{0} \\
\stackrel{0}{0}\end{array}$ & 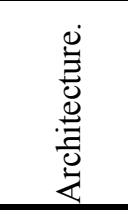 & 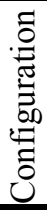 & 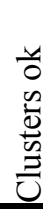 & 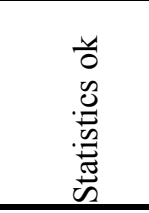 & 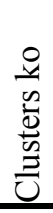 & 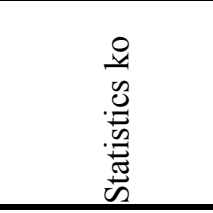 \\
\hline \multirow{8}{*}{8.8} & \multirow{4}{*}{3 screws } & 4 & 0 & - & 1 & 100 \\
\hline & & 3 & 2 & $16 / 6$ & 4 & $36 / 26 / 12 / 4$ \\
\hline & & 2 & 3 & $31 / 17 / 7$ & 3 & $25 / 17 / 3$ \\
\hline & & 1 & 3 & $21 / 6 / 1$ & 3 & $48 / 18 / 6$ \\
\hline & \multirow{4}{*}{4 screws } & 4 & 0 & - & 1 & 100 \\
\hline & & 3 & 3 & $48 / 16 / 6$ & 2 & $24 / 6$ \\
\hline & & 2 & 3 & $55 / 24 / 2$ & 2 & $16 / 3$ \\
\hline & & 1 & 3 & $49 / 19 / 5$ & 2 & $20 / 7$ \\
\hline
\end{tabular}


Depending on the failure risk of a configuration, specifications of the good directions or of the faulty ones could be given. For example, the third 4-screws configuration could be specified by the parameters variations of the first valid cluster that contains 48 variants because this specification encircles half the design space and would be so rather easy to follow. But it would be also possible to specify the bad direction that the designer has to avoid, especially for very risky configurations. These specifications could be given as parameter intervals to take into account in the design process and could be also visualized in a skeleton view as shown on figure 8 . The skeletons represent the shape of the simplified model used in the methodology; here the bigger valid cluster and the bigger faulty one are drawn. These figures are interesting to give the designer the outline of the design to create and allow having an idea of the global shape of the assembly. For example, in this case it should be better to avoid aligning the two junction beams of the lower bolts because these orientations may lead to failure, but it should be better to orient these beams towards the loading point. In the case of a 3 -screws solution that can be seen on figure 9, the main difference between the valid and the unacceptable clusters seem to be the section dimensions of the junction beams.

Finally, in order to make the good choice on this case, it would be interesting to have some comparisons of the costs between the different potential solutions; this aspect will be studied in further works. The final choice will depend on the trade-off that the designer wants to make: he has to choose between an expensive solution with few failure risks and a cheaper solution more difficult to design because subjected to several specifications in order to fulfil the numerous
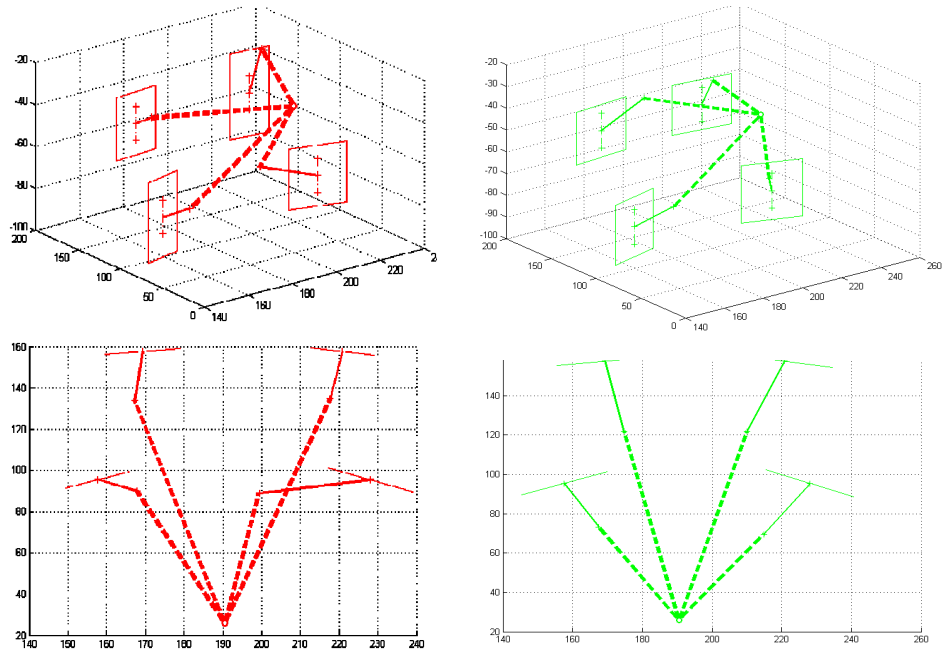

Figure 8: Visualization of the first bad (left) and good (right) clusters in perspective view (top) and in top view (bottom) for the third 4-screws configuration. 

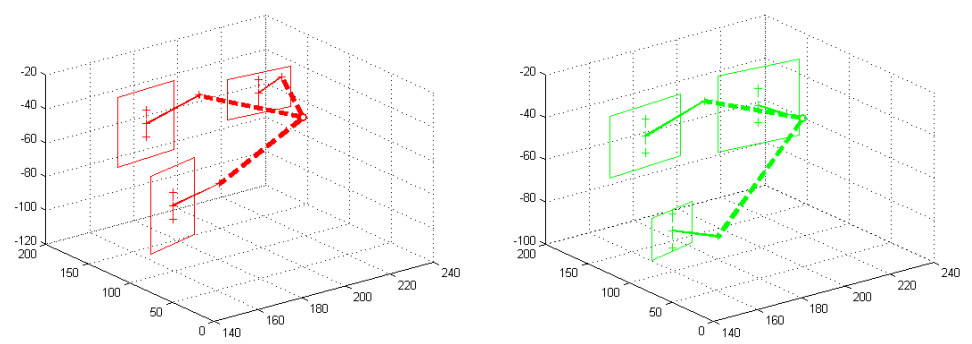

Figure 9: Visualisation of the first bad (left) and good (right) clusters in perspective view for the second 3 -screws configuration.

criteria. In this example, it is obvious that the 3-screws solutions are more hazardous than the 4-screws configurations, but depending on the compromise with the cost the designer wants to make, the choice will not be mechanically done for a 4-screws configuration.

\section{Conclusion}

This paper presents an innovative approach to quickly and efficiently choose the design of bolted joints in the first steps of the design process. The methodology is able to give several potential solutions with associated failure risks estimations and even some specifications in order to efficiently design a risky but cheaper configuration. An industrial application is presented to illustrate the useful results that can be given by the methodology developed.

This work allows giving very useful and relevant information with controlled risks when pre-dimensioning multi-bolted joints in industrial environment. Moreover, the reduced time necessary to obtain such results really contributes to decrease the design process duration without taking uncontrolled risks.

Some further work has to be done to improve the specifications phase, for example by using principal component analysis. Moreover, the overall methodology is tested by giving the results of the computation to designers to check if the outputs of the methodology presented in this paper are well interpreted for them to design the solutions. Then the methodology would be implemented in a design tool in order to be easily used by the designers.

\section{Acknowledgements}

This work has been performed within the framework of common researches between PSA Peugeot Citroën, LGMT (Laboratoire de Génie Mécanique de Toulouse) and LTDS (Laboratoire de Tribologie et Dynamique des Systèmes, Lyon). 


\section{References}

[1] Yannou B., Hamdi A. \& Landel E., A strategy for the conceptual modelling of vibro-acoustic performances in the preliminary design stage of a car subframe. Mécanique \& Industries, vol.4, pp. 365-376, 2003.

[2] Dericquebourg T., Daidié A., Debarle F. \& Sartor M., An original globallocal approach for predimensioning multi bolted joints. IDMME-Virtual Concept 2008, 2008.

[3] VDI Verein Deutscher Ingenieure, Systematic calculation of high duty bolted joints, joints with one cylindrical bolt, VDI 2230, VDI-Handbuch Konstruktion, Düsseldorf, 2003.

[4] Alliot J-M., Techniques d'optimisation stochastique appliquées à certains problèmes du trafic aérien, Le algorithmes génétiques (chapitre 1). http://www.recherche.enac.fr/opti/papers/thesis/HABIT/index.html

[5] Ke K., Li W. \& Sudjianto A., Algorithmic construction of optimal symmetric Latin hypercube designs. Journal of statistical Planning and Inference, 90, pp. 145-159, 2000.

[6] Prescott P., Orthogonal-column Latin hypercube designs with small samples, Computational Statistics and Data Analysis, 53, pp. 1191-1200, 2009. 